\title{
ANALYTICAL MODEL FOR INVESTIGATION OF THE EFFECT OF FRICTION POWER ON THERMAL STRESSES IN FRICTION ELEMENTS OF BRAKES
}

\author{
KATARZyNa TOPCZEWSKA \\ Bialystok University of Technology, Faculty of Mechanical Engineering, Bialystok, Poland \\ e-mail: k.topczewska@doktoranci.pb.edu.pl
}

\begin{abstract}
The influence of the change with time of friction power on thermal stresses in a friction element of a brake system is investigated. For this purpose, a list of ten different temporal profiles of specific friction power is used, which has been experimentally established for a single braking process. The corresponding profiles of transient temperature fields in the considered element are applied as input parameters to find quasi-static thermal stresses. Numerical analysis concerned with spatio-temporal distributions of the stresses and their evolutions on the heated surface is presented.
\end{abstract}

Keywords: frictional heating, temperature, specific power of friction, braking, thermal stresses

\section{Introduction}

One of the efficient and quick methods to preliminary estimate temperature and thermal stresses in a designed friction pair of a brake system is usage of analytical models. Generally, there are one-dimensional thermal problems of friction for bodies with canonical shape (i.e. semi-space, strip, circular disc), heated on its working surface by the heat flux with intensity proportional to the specific power of friction (Fazekas, 1953; Carslaw and Jaeger, 1959; Newcomb and Spurr, 1967). The last mentioned is defined as a product of the friction coefficient, contact pressure and relative sliding speed between the pad and the brake rotor (Blok, 1955; Ling, 1973). Experimental research shows that, in the real braking processes, the friction momentum (and hence the force of friction) vary with time, and the nature of this change depends on thermophysical properties of materials of the friction pair elements, working conditions and construction of the brake (Chichinadze et al., 1979; Balakin and Sergienko, 1999). As a result, the power of friction may have a complicated time profile. Most exact analytical solutions to the thermal problem of friction were obtained for constant or linearly decreasing with time specific power of friction (Yevtushenko and Kuciej, 2012; Kuciej, 2012; Jewtuszenko et al., 2015). Those solutions describe non-stationary fields of temperature in friction elements during braking with an invariable friction force and constant velocity or constant retardation. Distributions of thermal stresses initiated by such temperatures in a semi-space were investigated by Evtushenko and Kutsei (2006), and in a strip applied on the foundation analyzed by Yevtushenko et al. (2011), Yevtushenko and Kuciej (2010).

The list of different temporal profiles of the specific friction power, established by means of experimental research of frictional heating during single braking, was presented in the monograph by Chichinadze (1967). Exact, analytical solutions to boundary-value problems of heat conduction for a semi-space, heated on its surface by the frictional heat flux with intensity proportional to the specific friction power, placed on the above-mentioned list, were obtained in the articles by Topczewska (2017a) and Yevtushenko et al. (2017a). 
The aim of this paper is to investigate the influence of time profiles of the specific friction power on distributions of thermal stresses generated due to frictional heating in a friction element during single braking. Some results of the investigations were presented at the 9th International Conference Balttrib'2017 (Yevtushenko et al., 2017b).

\section{Temperature}

Temporal profiles of the specific friction power during single braking have the following forms (Chichinadze, 1967)

$$
q_{i}(t)=q_{0} q_{i}^{*}(t) \quad q_{0}=\frac{w_{0}}{t_{s}} \quad 0 \leqslant t \leqslant t_{s} \quad i=1,2, \ldots, 10
$$

where $t_{s}$ is the braking time, $q_{0}$ and $w_{0}$ are nominal values of the specific friction power and friction work, respectively

$$
\begin{array}{rlrl}
q_{1}^{*}(t) & =2\left(1-t^{*}\right) & q_{2}^{*}(t)=2 t^{*} & q_{3}^{*}(t)=1.5 \sqrt{1-t^{*}} \\
q_{4}^{*}(t) & =1.5 \sqrt{t^{*}} & q_{5}^{*}(t)=3\left(1-t^{*}\right)^{2} & q_{6}^{*}(t)=3 t^{* 2} \\
q_{7}^{*}(t) & =6 t^{*}\left(1-t^{*}\right) & q_{8}^{*}(t)=1.2\left(1-t^{*}\right)\left(1+2 t^{*}\right) \\
q_{9}^{*}(t) & =1.2 t^{*}\left(3-2 t^{*}\right) & q_{10}^{*}(t)=6 \sqrt{t^{*}}\left(1-\sqrt{t^{*}}\right) & t^{*}=\frac{t}{t_{s}}
\end{array}
$$

where

$$
w_{0}=\int_{0}^{t_{s}} q_{i}(t) d t \quad i=1,2, \ldots, 10
$$

is constant for all cases, at the end of braking $t=t_{s}$.

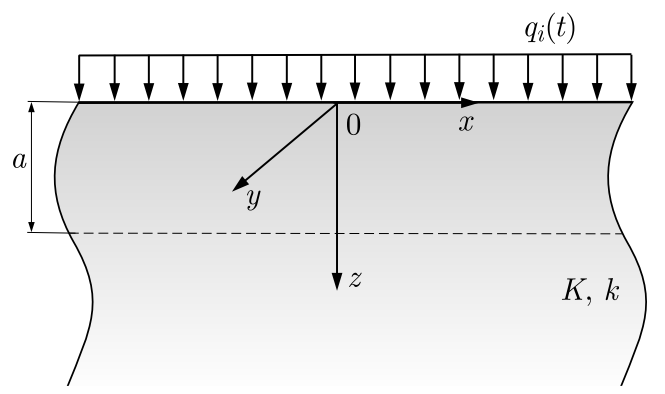

Fig. 1. Scheme of the problem

Temperature fields $T_{i}(z, t), z \geqslant 0,0 \leqslant t \leqslant t_{s}, i=1,2, \ldots, 10$ in a friction element (pad, disc, etc.) caused by heating its working surface (Fig. 1) by the heat flux with intensities $q_{i}(t)$, $i=1,2, \ldots, 10(2.1)-(2.3)$ are found from exact solutions to the proper thermal problem of friction for a semi-space $z \geqslant 0$ in dimensionless forms (Topczewska, 2017a; Yevtushenko et al., 2017a) 


$$
\begin{aligned}
& T_{1}^{*}(\zeta, \tau)=\frac{4}{3} \sqrt{\tau} \tau^{*}\left\{\left[\frac{3}{\tau^{*}}-2\left(1+Z^{2}\right)\right] \operatorname{ierfc} Z+Z \operatorname{erfc} Z\right\} \\
& T_{2}^{*}(\zeta, \tau)=\frac{4}{3} \sqrt{\tau} \tau^{*}\left[2\left(1+Z^{2}\right) \operatorname{ierfc} Z-Z \operatorname{erfc} Z\right] \\
& T_{3}^{*}(\zeta, \tau)=3 \sqrt{\tau} \operatorname{ierfc} Z-\frac{1}{2} \sqrt{\tau} \tau^{*}\left[2\left(1+Z^{2}\right) \operatorname{ierfc} Z-Z \operatorname{erfc} Z\right] \\
& \quad-\frac{1}{40} \sqrt{\tau} \tau^{* 2}\left[\left(8+18 Z^{2}+4 Z^{4}\right) \operatorname{ierfc} Z-Z\left(7+2 Z^{2}\right) \operatorname{erfc} Z\right] \\
& \quad-\frac{1}{560} \sqrt{\tau} \tau^{* 3}\left[\left(48+174 Z^{2}+80 Z^{4}+8 Z^{6}\right) \operatorname{ierfc} Z-Z\left(57+36 Z^{2}+4 Z^{4}\right) \operatorname{erfc} Z\right] \\
& T_{4}^{*}(\zeta, \tau)=\frac{3}{4} \sqrt{\pi \tau_{s}} \tau^{*}[\operatorname{erfc} Z-2 Z \operatorname{ierfc} Z] \\
& T_{5}^{*}(\zeta, \tau)=2 \sqrt{\tau}\left\{3 \operatorname{ierfc} Z-2 \tau^{*}\left[2\left(1+Z^{2}\right) \operatorname{ierfc} Z-Z \operatorname{erfc} Z\right]\right. \\
& \left.\quad+\frac{1}{5} \tau^{* 2}\left[\left(8+18 Z^{2}+4 Z^{4}\right) \operatorname{ierfc} Z-Z\left(7+2 Z^{2}\right) \operatorname{erfc} Z\right]\right\} \\
& T_{6}^{*}(\zeta, \tau)=\frac{2}{5} \sqrt{\tau} \tau^{* 2}\left[\left(8+18 Z^{2}+4 Z^{4}\right) \operatorname{ierfc} Z-Z\left(7+2 Z^{2}\right) \operatorname{erfc} Z\right] \\
& T_{7}^{*}(\zeta, \tau)=4 \sqrt{\tau} \tau^{*}\left\{\left[2\left(1+Z^{2}\right) \operatorname{ierfc} Z-Z \operatorname{erfc} Z\right]\right. \\
& \left.\quad-\frac{1}{5} \tau^{*}\left[\left(8+18 Z^{2}+4 Z^{4}\right) \operatorname{ierfc} Z-Z\left(7+2 Z^{2}\right) \operatorname{erfc} Z\right]\right\} \\
& T_{8}^{*}(\zeta, \tau)=\frac{4}{5} \sqrt{\tau}\left\{3 \operatorname{ierfc} Z+\tau^{*}\left[2\left(1+Z^{2}\right) \operatorname{ierfc} Z-Z \operatorname{erfc} Z\right]\right. \\
& \left.\quad-\frac{2}{5} \tau^{* 2}\left[\left(8+18 Z^{2}+4 Z^{4}\right) \operatorname{ierfc} Z-Z\left(7+2 Z^{2}\right) \operatorname{erfc} Z\right]\right\} \\
& T_{9}^{*}(\zeta, \tau)=\frac{4}{5} \sqrt{\tau} \tau^{*}\left\{3\left[2\left(1+Z^{2}\right) \operatorname{ierfc} Z-Z \operatorname{erfc} Z\right]\right. \\
& \left.-\frac{2}{5} \tau^{*}\left[\left(8+18 Z^{2}+4 Z^{4}\right) \operatorname{ierfc} Z-Z\left(7+2 Z^{2}\right) \operatorname{erfc} Z\right]\right\} \\
& T_{1} 0^{*}(\zeta, \tau)=\tau^{*}\left\{3 \sqrt{\pi \tau_{s}}(\operatorname{erfc} Z-2 Z \operatorname{ierfc} Z)-4 \sqrt{\tau}\left[2\left(1+Z Z^{2}\right) \operatorname{ierfc} Z-Z \operatorname{erfc} Z\right]\right\}
\end{aligned}
$$

where the function $\operatorname{erf}(x)$ is error function, $\operatorname{erfc}(x)=1-\operatorname{erf}(x), \operatorname{ierfc}(x)=\exp \left(-x^{2}\right) / \sqrt{\pi}-$ $x \operatorname{erfc}(x)$, and the dimensionless parameters are determined by the following relations

$$
\begin{array}{lcc}
\zeta=\frac{z}{a} & \tau=\frac{k t}{a^{2}} & \tau_{s}=\frac{k t_{s}}{a^{2}} \quad \tau^{*}=\frac{\tau}{\tau_{s}} \\
Z=\frac{\zeta}{2 \sqrt{\tau}} & T_{0}=\frac{q_{0} a}{K} & T_{i}^{*}=\frac{T_{i}-T_{a}}{T_{0}}
\end{array}
$$

and $a=\sqrt{3 k t_{s}}$ - effective depth of heat penetration (Chichinadze et al., 1979), $K$ - thermal conductivity $[\mathrm{W} /(\mathrm{Km})] ; k$ - thermal diffusivity $\left[\mathrm{m}^{2} / \mathrm{s}\right] ; T_{a}$ - ambient temperature $[\mathrm{K}]$.

\section{Thermal stresses}

Thermal stresses corresponding to the fields of temperature $T_{i}^{*}(\zeta, \tau)$, Eqs. (2.4) and (2.5), are found based on the model of thermal bending of a thick plate with unfixed ends. In accordance with this model, $\sigma_{i, x}(z, t)=\sigma_{i, y}(z, t) \equiv \sigma_{i}(z, t), \sigma_{i, z}(z, t)=0$, where the transverse normal component of the stress tensor $\sigma_{i}(i=1,2, \ldots, 10)$ (Noda et al., 2000)

$$
\sigma_{i}(z, t)=\sigma_{0} \sigma_{i}^{*}(\zeta, \tau) \quad \sigma_{0}=\frac{\alpha_{t} E T_{0}}{1-\nu} \quad 0 \leqslant z \leqslant a \quad 0 \leqslant t \leqslant t_{s}
$$


where the material properties are $E$ - Young's modulus [MPa], $\alpha_{t}$-coefficient of linear thermal expansion $\left[\mathrm{K}^{-1}\right], \nu$ - Poisson's ratio

$$
\begin{array}{ll}
\sigma_{i}^{*}(\zeta, \tau)=\varepsilon_{i}^{*}(\zeta, \tau)-T_{i}^{*}(\zeta, \tau) & 0 \leqslant \zeta \leqslant 1 \\
\varepsilon_{i}^{*}(\zeta, \tau)=(4-6 \zeta) N_{i}(\tau)+6(2 \zeta-1) M_{i}(\tau) & 0 \leqslant \tau \leqslant \tau_{s}
\end{array}
$$

and

$$
N_{i}(\tau)=\int_{0}^{1} T_{i}^{*}(\zeta, \tau) d \zeta \quad M_{i}(\tau)=\int_{0}^{1} \zeta T_{i}^{*}(\zeta, \tau) d \zeta
$$

Substituting the dimensionless temperatures $T_{i}^{*}(\zeta, \tau)$, Eq. (2.4) to equations (3.3), the following are determined

$$
\begin{aligned}
& N_{1}(\tau)=\frac{8}{3} \tau \tau^{*}\left[\left(\frac{3}{\tau^{*}}-2\right) I_{0}(\tau)-2 I_{2}(\tau)+J_{1}(\tau)\right] \\
& M_{1}(\tau)=\frac{16}{3} \tau \sqrt{\tau} \tau^{*}\left[\left(\frac{3}{\tau^{*}}-2\right) I_{1}(\tau)-2 I_{3}(\tau)+J_{2}(\tau)\right] \\
& N_{2}(\tau)=\frac{8}{3} \tau \tau^{*}\left\{2\left[I_{0}(\tau)+I_{2}(\tau)\right]-J_{1}(\tau)\right\} \\
& M_{2}(\tau)=\frac{16}{3} \tau \sqrt{\tau} \tau^{*}\left\{2\left[I_{1}(\tau)+I_{3}(\tau)\right]-J_{2}(\tau)\right\} \\
& N_{3}(\tau)=6 \tau I_{0}(\tau)-\tau \tau^{*}\left\{2\left[I_{0}(\tau)+I_{2}(\tau)\right]-J_{1}(\tau)\right\} \\
& -\frac{1}{20} \tau \tau^{* 2}\left[8 I_{0}(\tau)+18 I_{2}(\tau)+4 I_{4}(\tau)-7 J_{1}(\tau)-2 J_{3}(\tau)\right] \\
& -\frac{1}{280} \tau \tau^{* 3}\left[48 I_{0}(\tau)+174 I_{2}(\tau)+80 I_{4}(\tau)+8 I_{6}(\tau)-57 J_{1}(\tau)-36 J_{3}(\tau)-4 J_{5}(\tau)\right] \\
& M_{3}(\tau)=12 \tau \sqrt{\tau} I_{1}(\tau)-2 \tau \sqrt{\tau} \tau^{*}\left\{2\left[I_{1}(\tau)+I_{3}(\tau)\right]-J_{2}(\tau)\right\} \\
& -\frac{1}{10} \tau \sqrt{\tau} \tau^{* 2}\left[8 I_{1}(\tau)+18 I_{3}(\tau)+4 I_{5}(\tau)-7 J_{2}(\tau)-2 J_{4}(\tau)\right] \\
& -\frac{1}{140} \tau \sqrt{\tau} \tau^{* 3}\left[48 I_{1}(\tau)+174 I_{3}(\tau)+80 I_{5}(\tau)+8 I_{7}(\tau)-57 J_{2}(\tau)-36 J_{4}(\tau)-4 J_{6}(\tau)\right] \\
& N_{4}(\tau)=\frac{3}{2} \sqrt{\pi \tau \tau_{s}} \tau^{*}\left[J_{0}(\tau)-2 I_{1}(\tau)\right] \quad M_{4}(\tau)=3 \tau \sqrt{\pi \tau_{s}} \tau^{*}\left[J_{1}(\tau)-2 I_{2}(\tau)\right] \\
& N_{5}(\tau)=4 \tau\left\{3 I_{0}(\tau)-2 \tau^{*}\left[2 I_{0}(\tau)+2 I_{2}(\tau)-J_{1}(\tau)\right]\right. \\
& \left.+\frac{1}{5} \tau^{* 2}\left[8 I_{0}(\tau)+18 I_{2}(\tau)+4 I_{4}(\tau)-7 J_{1}(\tau)-2 J_{3}(\tau)\right]\right\} \\
& M_{5}(\tau)=8 \tau \sqrt{\tau}\left\{3 I_{1}(\tau)-2 \tau^{*}\left[2 I_{1}(\tau)+2 I_{3}(\tau)-J_{2}(\tau)\right]\right. \\
& \left.+\frac{1}{5} \tau^{* 2}\left[8 I_{1}(\tau)+18 I_{3}(\tau)+4 I_{5}(\tau)-7 J_{2}(\tau)-2 J_{4}(\tau)\right]\right\} \\
& N_{6}(\tau)=\frac{4}{5} \tau \tau^{* 2}\left[8 I_{0}(\tau)+18 I_{2}(\tau)+4 I_{4}(\tau)-7 J_{1}(\tau)-2 J_{3}(\tau)\right] \\
& M_{6}(\tau)=\frac{8}{5} \tau \sqrt{\tau} \tau^{* 2}\left[8 I_{1}(\tau)+18 I_{3}(\tau)+4 I_{5}(\tau)-7 J_{2}(\tau)-2 J_{4}(\tau)\right] \\
& N_{7}(\tau)=8 \tau \tau^{*}\left\{2 I_{0}(\tau)+2 I_{2}(\tau)-J_{1}(\tau)\right. \\
& \left.-0.2 \tau^{*}\left[8 I_{0}(\tau)+18 I_{2}(\tau)+4 I_{4}(\tau)-7 J_{1}(\tau)-2 J_{3}(\tau)\right]\right\} \\
& M_{7}(\tau)=16 \tau \sqrt{\tau} \tau^{*}\left\{2 I_{1}(\tau)+2 I_{3}(\tau)-J_{2}(\tau)\right. \\
& \left.-\frac{1}{5} \tau^{*}\left[8 I_{1}(\tau)+18 I_{3}(\tau)+4 I_{5}(\tau)-7 J_{2}(\tau)-2 J_{4}(\tau)\right]\right\}
\end{aligned}
$$




$$
\begin{aligned}
& N_{8}(\tau)=\frac{8}{5} \tau\left\{3 I_{0}(\tau)+\tau^{*}\left[2 I_{0}(\tau)+2 I_{2}(\tau)-J_{1}(\tau)\right]\right. \\
& \left.-\frac{2}{5} \tau^{* 2}\left[8 I_{0}(\tau)+18 I_{2}(\tau)+4 I_{4}(\tau)-7 J_{1}(\tau)-2 J_{3}(\tau)\right]\right\} \\
& M_{8}(\tau)=\frac{16}{5} \tau \sqrt{\tau}\left\{3 I_{1}(\tau)+\tau^{*}\left[2 I_{1}(\tau)+2 I_{3}(\tau)-J_{2}(\tau)\right]\right. \\
& \left.-\frac{2}{5} \tau^{* 2}\left[8 I_{1}(\tau)+18 I_{3}(\tau)+4 I_{5}(\tau)-7 J_{2}(\tau)-2 J_{4}(\tau)\right]\right\} \\
& N_{9}(\tau)=\frac{8}{5} \tau \tau^{*}\left\{3\left[2 I_{0}(\tau)+2 I_{2}(\tau)-J_{1}(\tau)\right]\right. \\
& \left.-\frac{2}{5} \tau^{*}\left[8 I_{0}(\tau)+18 I_{2}(\tau)+4 I_{4}(\tau)-7 J_{1}(\tau)-2 J_{3}(\tau)\right]\right\} \\
& M_{9}(\tau)=\frac{1}{5} \tau \sqrt{\tau} \tau^{*}\left\{3\left[2 I_{1}(\tau)+2 I_{3}(\tau)-J_{2}(\tau)\right]\right. \\
& \left.-\frac{2}{5} \tau^{*}\left[8 I_{1}(\tau)+18 I_{3}(\tau)+4 I_{5}(\tau)-7 J_{2}(\tau)-2 J_{4}(\tau)\right]\right\} \\
& N_{10}(\tau)=2 \sqrt{\tau} \tau^{*}\left\{3 \sqrt{\pi \tau_{s}}\left[J_{0}(\tau)-2 I_{1}(\tau)\right]-8 \sqrt{\tau}\left[I_{0}(\tau)+I_{2}(\tau)-\frac{1}{2} J_{1}(\tau)\right]\right\} \\
& M_{10}(\tau)=4 \tau \tau^{*}\left\{3 \sqrt{\pi \tau_{s}}\left[J_{1}(\tau)-2 I_{2}(\tau)\right]-8 \sqrt{\tau}\left[I_{1}(\tau)+I_{3}(\tau)-\frac{1}{2} J_{2}(\tau)\right]\right\}
\end{aligned}
$$

where

$$
\begin{array}{lr}
I_{k}(\tau)=\int_{0}^{X} Z^{k} \operatorname{ierfc} Z d Z=\frac{1}{\sqrt{\pi}} L_{k}(\tau)-J_{k+1}(\tau) \quad k=0,1, \ldots, 7 \\
L_{k}(\tau)=\int_{0}^{X} Z^{k} \mathrm{e}^{-Z^{2}} d Z & J_{k}(\tau)=\int_{0}^{X} Z^{k} \operatorname{erfc} Z d Z
\end{array}
$$

and the variable $Z$ has the form of Eq. (2.5).

Using the recursive formulas (Prudnikov et al., 1986, 1998), $k=2,3, \ldots$

$$
\begin{aligned}
& L_{k}(\tau)=\frac{1}{2}\left[(k-1) L_{k-2}(\tau)-X^{k-1} \mathrm{e}^{-X^{2}}\right] \\
& J_{k}(\tau)=\frac{1}{k+1}\left[\frac{1}{2} k(k-1) J_{k-2}(\tau)+\left(X^{2}-\frac{1}{2} k\right) X^{k-1} \operatorname{erfc} X-\frac{1}{\sqrt{\pi}} X^{k} \mathrm{e}^{-X^{2}}\right]
\end{aligned}
$$

and taking into account that

$$
\begin{array}{rlrl}
L_{0}(\tau) & =\frac{1}{2} \sqrt{\pi}(1-\operatorname{erfc} X) & L_{1}(\tau) & =\frac{1}{2}\left(1-\mathrm{e}^{-X^{2}}\right) \\
J_{0}(\tau) & =\frac{1}{\sqrt{\pi}}-\operatorname{ierfc} X & J_{1}(\tau)=\frac{1}{4} \operatorname{erf} X-\frac{1}{2} X \text { ierfc } X
\end{array}
$$

integrals $(3.5)_{2}$ are computed as

$$
\begin{aligned}
& L_{2}(\tau)=\frac{1}{4} \sqrt{\pi} \operatorname{erf} X-\frac{1}{2} X \mathrm{e}^{-X^{2}} \quad L_{3}(\tau)=\frac{1}{2}\left[1-\left(1+X^{2}\right) \mathrm{e}^{-X^{2}}\right] \\
& L_{4}(\tau)=0.375 \sqrt{\pi} \operatorname{erf} X-\frac{1}{2} X\left(X^{2}+\frac{3}{2}\right) \mathrm{e}^{-X^{2}} \\
& L_{5}(\tau)=1-\left(\frac{1}{2} X^{4}+X^{2}+1\right) \mathrm{e}^{-X^{2}} \\
& L_{6}(\tau)=0.9375 \sqrt{\pi} \operatorname{erf} X-X\left(0.5 X^{4}+1.25 X^{2}+1.875\right) \mathrm{e}^{-X^{2}} \\
& L_{7}(\tau)=3-\left(\frac{1}{2} X^{6}+\frac{3}{2} X^{4}+3 X^{2}+3\right) \mathrm{e}^{-X^{2}}
\end{aligned}
$$


and

$$
\begin{aligned}
& J_{2}(\tau)=\frac{1}{3}\left\{X^{3} \operatorname{erfc} X+\frac{1}{\sqrt{\pi}}\left[1-\left(X^{2}+1\right) \mathrm{e}^{-X^{2}}\right]\right\} \\
& \left.J_{3}(\tau)=\frac{1}{4}\left[X^{4} \operatorname{erfc} X+\frac{3}{4} \operatorname{erf} X-\frac{1}{\sqrt{\pi}} X\left(X^{2}+\frac{3}{2}\right) \mathrm{e}^{-x^{2}}\right)\right] \\
& J_{4}(\tau)=\frac{1}{5}\left\{X^{5} \operatorname{erfc} X+\frac{1}{\sqrt{\pi}}\left[2-\left(X^{4}+2 X^{2}+2\right) \mathrm{e}^{-X^{2}}\right]\right\} \\
& J_{5}(\tau)=\frac{1}{6}\left[X^{6} \operatorname{erfc} X+1.875 \operatorname{erf} X-\frac{1}{\sqrt{\pi}} X\left(X^{4}+2.5 X^{2}+3.75\right) \mathrm{e}^{-X^{2}}\right] \\
& J_{6}(\tau)=\frac{1}{7}\left\{X^{7} \operatorname{erfc} X+\frac{1}{\sqrt{\pi}}\left[6-\left(X^{6}+3 X^{4}+6 X^{2}+6\right) \mathrm{e}^{-X^{2}}\right]\right\} \\
& J_{7}(\tau)=\frac{1}{8}\left[X^{8} \operatorname{erfc} X+6.5625 \operatorname{erf} X-\frac{1}{\sqrt{\pi}} X\left(X^{6}+3.5 X^{4}+8.75 X^{2}+13.125\right) \mathrm{e}^{-X^{2}}\right] \\
& J_{8}(\tau)=\frac{1}{9}\left\{X^{9} \operatorname{erfc} X+\frac{1}{\sqrt{\pi}}\left[24-\left(X^{8}+4 X^{6}+12 X^{4}+24 X^{2}+24\right) \mathrm{e}^{-X^{2}}\right]\right\}
\end{aligned}
$$

With account of relations (3.7)-(3.9), in the right side of equation $(3.5)_{1}$, the following expressions are obtained

$$
\begin{aligned}
& I_{0}(\tau)=\frac{1}{2 \sqrt{\pi}} X \mathrm{e}^{-X^{2}}+\frac{1}{4} \operatorname{erf} X-\frac{1}{2} X^{2} \operatorname{erfc} X \\
& I_{1}(\tau)=\frac{1}{6}\left\{\frac{1}{\sqrt{\pi}}\left[1+\left(2 X^{2}-1\right) \mathrm{e}^{-X^{2}}\right]-2 X^{3} \operatorname{erfc} X\right\} \\
& I_{2}(\tau)=\frac{1}{8}\left[X\left(2 X^{2}-1\right) \mathrm{e}^{-X^{2}} \sqrt{\pi}+\frac{1}{2} \operatorname{erf} X-2 X^{4} \operatorname{erfc} X\right] \\
& I_{3}(\tau)=\frac{1}{5}\left\{\frac{1}{2 \sqrt{\pi}}\left[1+\left(2 X^{4}-X^{2}-1\right) \mathrm{e}^{-X^{2}}\right]-X^{5} \operatorname{erfc} X\right. \\
& I_{4}(\tau)=\frac{1}{6}\left[\frac{1}{4 \sqrt{\pi}} X\left(4 X^{4}-2 X^{2}-3\right) \mathrm{e}^{-X^{2}}+0.375 \operatorname{erf} X-X^{6} \operatorname{erfc} X\right] \\
& I_{5}(\tau)=\frac{1}{7}\left\{\frac{1}{\sqrt{\pi}}\left[1+\left(X^{6}-\frac{1}{2} X^{4}-X^{2}-1\right) \mathrm{e}^{-X^{2}}\right]-X^{7} \operatorname{erfc} X\right\} \\
& I_{6}(\tau)=\frac{1}{8}\left\{\frac{1}{\sqrt{\pi}} X\left(X^{6}-0.5 X^{4}-1.25 X^{2}-1.875\right) \mathrm{e}^{-X^{2}}+0.9375 \operatorname{erf} X-X^{8} \operatorname{erfc} X\right\} \\
& I_{7}(\tau)=\frac{1}{9}\left\{\frac{1}{\sqrt{\pi}}\left[3+\left(X^{8}-\frac{1}{2} X^{6}-\frac{3}{2} X^{4}-3 X^{2}-3\right) \mathrm{e}^{-X^{2}}\right]-X^{9} \operatorname{erfc} X\right\}
\end{aligned}
$$

Substituting functions $J_{k}(\tau), I_{k}(\tau), k=0,1, \ldots, 7$, Eqs. $(3.7)_{2},(3.9)$ and (3.10) to Eqs. (3.4), temporal profiles of temperature $N_{i}(\tau)$ averaged over the plate thickness and the temperature momentum $M_{i}(\tau), i=1,2, \ldots, 10$, Eqs. (3.3), and next, dimensionless stresses $\sigma_{i}^{*}(\zeta, \tau)$, Eqs. (3.2), have been determined.

It should be noted that distributions of the thermal stresses corresponding to the profiles of the specific friction power $q_{i}^{*}(t), i=5,7,10$, Eqs. (2.2), were studied in the article by Topczewska (2017b), too.

\section{Numerical analysis}

Influence of the temporal profiles of the specific friction power $q_{i}^{*}(t), i=1,2, \ldots, 10$, Eqs. (2.2) on the dimensionless temperature $T_{i}^{*}(\zeta, \tau)(2.4)$ was detailed investigated in the article by Yevtushenko et al. (2017a). Therefore, in this paper, the effect of this factor on the quasi-static normal thermal stresses $\sigma_{i}^{*}(\zeta, \tau), i=1,2, \ldots, 10$ Eqs. (3.1) and (3.2), initiated by the 
temperature, have been determined. Isolines of these stresses are shown in Figs. 2 and 3. The presented data can be divided into two specific groups. The first one consists of the results obtained for $\mathrm{i}=1,3,8$ and $\mathrm{i}=5,7,10$ (Topczewska, 2017b). These cases are characterized by the fact that with the onset of the braking process, along with a rapid increase of temperature, in the region $0 \leqslant \zeta \leqslant 0.2$ under the friction surface, compressive stresses $\left(\sigma_{i}^{*}<0\right)$ appear. Absolute values of the stresses in this zone decrease with time, achieving zero at the particular time moment, then change the sign and become tensile stresses $\left(\sigma_{i}^{*}>0\right)$. The time of change of the stress sing (compressive into tensile) is strongly dependent on the time of achieving the maximum temperature on the friction surface of the heated element. The faster temperature reaches the maximum value, the earlier this transition occurs and the tensile stress achieves a higher value on the outer surface at the moment of standstill. The second region $0.75 \leqslant \zeta \leqslant 1$ of the compressive stresses occurs adjacent to the unheated, bottom surface of the element.

Evolution of the stresses in this region is similar, as in the previous - at the initial stage
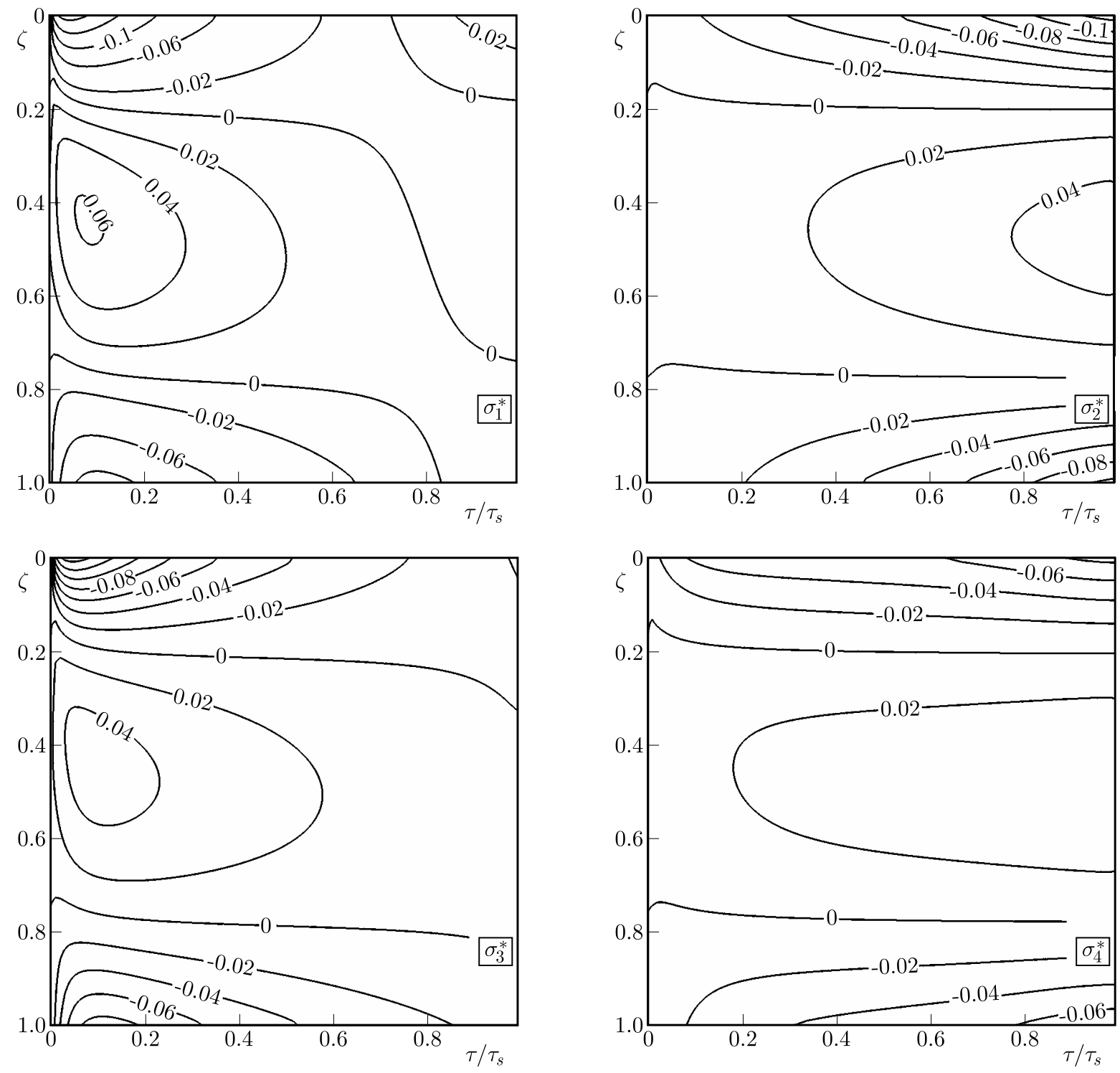

Fig. 2. Isolines of the dimensionless normal transverse stresses $\sigma_{i}^{*}, i=1,2,3,4$

of braking the compressive stresses appear, then change their sign with approaching to the stop moment. Between these zones of tensile stresses, there is a region of compressive stresses, which rapidly increase at the initial stage of braking, achieving their maximum values after 

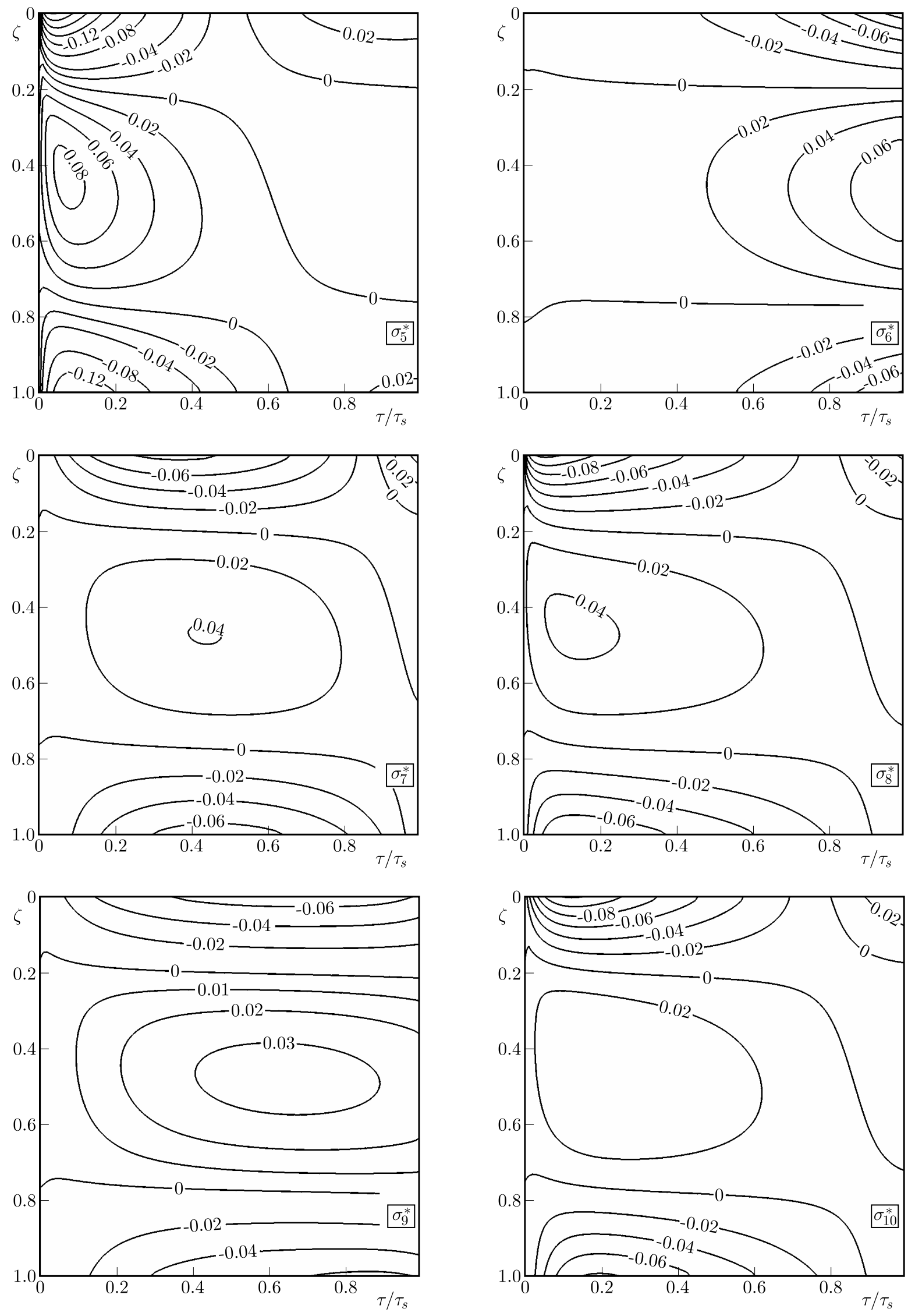

Fig. 3. Isolines of the dimensionless normal transverse stresses $\sigma_{i}^{*}, i=5,6,7,8,9,10$ 
a relatively short time. Subsequently, these stresses decrease with time, disappearing near the moment of standstill. Values and evolutions of the stresses in the all above-mentioned regions are interdependent. The higher values of tensile stresses on the surface occurs, the higher values of the compressive stresses are achieved. The time of occurrence of the maximum tensile stresses inside the friction element is almost equal to the time of appearance of the greatest values of compressive stresses on its heated surface.

Stresses isolines $\sigma_{i}^{*}(\zeta, \tau), i=2,4,6,9$, from the second group, reflect a monotonical increase of the corresponding temperatures $T_{i}^{*}(\zeta, \tau)$ during braking with set distance from the heated surface $\zeta$ (Yevtushenko et al., 2017a). In contrast to the first group, in the considered regions of the greatest concentration of compressive stresses, adjacent to the upper and lower edge of the friction element, the maxima are located closer to the end of the braking process, and the maximum absolute values on the friction surface $\zeta=0$ are achieved at the stop moment $\tau=\tau_{s}$. In this second group, between the lines of zero stresses, which remain during the whole braking process at depths $\zeta \approx 0.2$ and $\zeta \approx 0.75$, also the region of tensile stresses occurs. These stresses monotonically increase with time, reaching the maximum value at the stop moment.

(a)

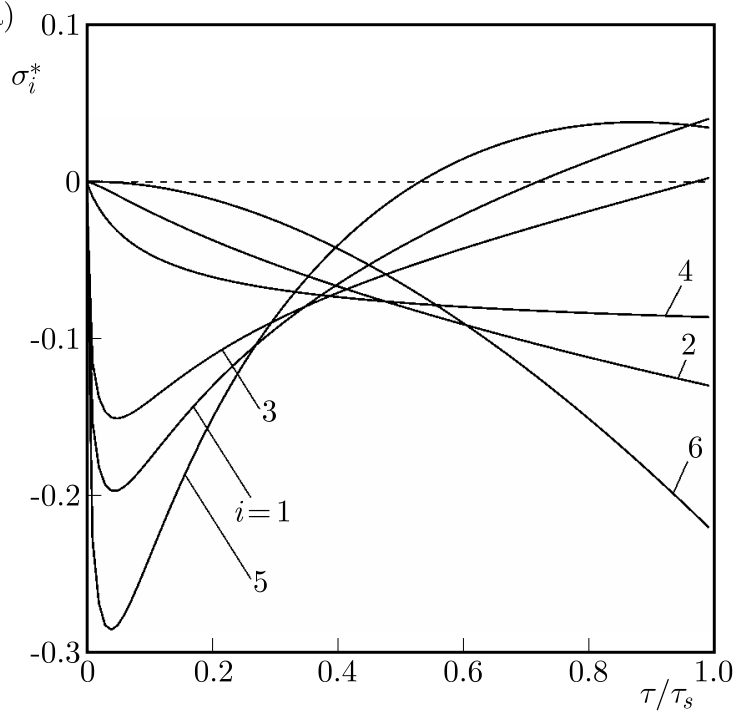

(b)

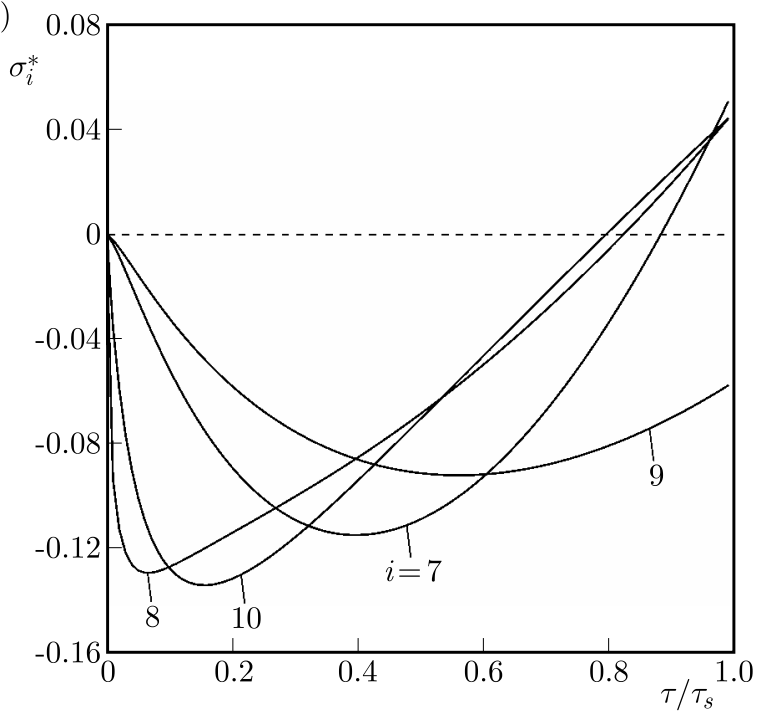

Fig. 4. Evolutions of the dimensionless normal transverse stresses $\sigma_{i}^{*}$ on the surface of friction $\zeta=0$ : (a) $i=1,2, \ldots, 6$; (b) $i=7,8,9,10$

Changes of the dimensionless thermal stresses $\sigma_{i}^{*}(0, \tau), i=1,2, \ldots, 10$ with time on the friction surface of the element are presented in Fig. 4. As shown in the article by Yevtushenko et al. (2017a), temporal profiles of the specific friction power $q_{i}^{*}(\tau), i=1,2, \ldots, 10(2.1)-(2.3)$ can be classified into one of three specific groups. The first group includes functions $q_{i}^{*}(t), i=1,3,5$, which reach the maximum value at the initial moment of braking, and then monotonically decrease to zero at the standstill moment. The corresponding stresses on the heated surface, after start of the process are compressive, and their absolute values rapidly increase, reaching the maximum values $0.2,0.15$ and 0.29 at moments $0.04 \tau_{s}, 0.05 \tau_{s}$ and $0.04 \tau_{s}$ for $i=1,3,5$, accordingly (Fig. 4a). Then, the compressive stresses on the heated surface disappear with time, and at the moments $0.72 \tau_{s}(i=1), 0.97 \tau_{s}(i=3)$ and $0.53 \tau_{s}(i=5)$ change the sign of stresses, and the tensile stresses appear.

The second group contains the functions $q_{i}^{*}(t), i=2,4,6$ (2.1) and (2.2), which monotonically increase from zero at the initial moment of the braking process to the maximum value at the stop moment. In a similar way, the evolutions of the corresponding dimensionless temperatures $T_{i}^{*}$, $i=2,4,6$ are calculated on the friction surface $\zeta=0$ from equations $(2.4)_{2},(2.4)_{4}$ and $(2.4)_{6}$, 
respectively. Dimensionless thermal stresses $\sigma_{i}^{*}, i=2,4,6$ on the heated surface are compressed during the whole braking, and their absolute values monotonically increase with time from zero at the start moment to the maximum values $0.13(i=2), 0.09(i=4), 0.22(i=6)$, at the standstill (Fig. 4a).

The last one of the three highlighted groups consists of four time profiles of the specific friction power $q_{i}^{*}(t), i=7,8,9,10$, which have a local maximum within the time braking interval. Profiles of these functions affect the evolution of the corresponding stresses. At the initial stage of braking, the absolute values of compressive stresses decrease, reaching a local minimum -0.16 at $\tau=0.4 \tau_{s}(i=7),-0.13$ at $\tau=0.07 \tau_{s}(i=8),-0.09$ at $\tau=0.56 \tau_{s}(i=9)$ and -0.13 at $\tau=0.16 \tau_{s}(i=10)$ (Fig. 4b). Stresses $\sigma_{i}^{*}, i=7,8,10$ at the moments $\tau=0.88, \tau=0.82$ and $\tau=0.8$, accordingly, change the sign, and their highest values $0.05,0.044$ and 0.044 are achieved at the stop moment. In the case $i=9$, stresses on the friction surface of the element compress the material during the whole braking process.

\section{Conclusions}

It is known that the sum of the intensities of heat fluxes generated due to friction on the contact surface of the friction pair (which are directed along the normal to the inside of the friction elements) are equal to the specific friction power (Ling, 1973). Changes of friction power with time can be different, and their typical cases were classified in the monograph by Chichinadze et al. (1967). Using this classification, in the articles by Topczewska (2017a) and Yevtushenko et al. (2017a) it has been established that the temporal profile of the specific friction power has crucial influence on the distribution of temperature in the pad and the brake disk, and the maximum value of temperature on the contact surface of these elements. In this paper, based on exact analytical solutions to the boundary-value problem of heat conduction for a semi-space heated on its surface by the heat flux with intensity equal to the specific power of friction, the exact solution of the boundary quasi-static problems of thermoelasticity has been received. This allows one to conduct numerical analysis and to investigate the influence of the time profile of friction power on the state of thermal stresses in a selected element of the friction pair. As a result, it has been established that the values and distribution of the thermal stresses initiated by frictional heating depends mainly on the temporal profile of a specific friction power. If the friction power monotonically increases during braking $(i=2,4,6)$ or its maximum value is reached near the moment of standstill $(i=9)$, on the friction surface of the element there occur merely compressive normal stresses. However, when the maximum values of the friction power and also temperature are achieved earlier during the braking process $(i=1,3,5,7,8,10)$, then due to relative cooling of the surface, before the standstill moment, the stresses change the sign and tensile stresses appear. In the case of exceeding the ultimate strength of the friction material by the value of this stress, initiation of the superficial thermal cracks may appear (Evtushenko and Kutsei, 2006; Yevtushenko et al., 2011).

It has been established that the relationship between the maximum values of specific friction power and the normal transverse stresses is directly proportional. However, the moment of thermal stresses change of sign on the friction surface (transition from compressive to tensile stresses) mainly depend on the time of achieving the maximum temperature. The most rational modes of frictional heating from the "stresses" point of view corresponds the following temporal profiles $q_{i}^{*}(t), i=2,4$ and 9 . For the same brake work, evolutions of the stresses corresponding to these time profiles are the most uniform and the change of their sign does not occur. Their extreme values are the lowest in comparison with other cases. 


\section{References}

1. Balakin V. A., Sergienko V. P., 1999, Heat Calculations of Brakes and Friction Units (in Russian), MPRI of NASB, Gomel,

2. Blok H., 1955, The dissipation of friction heat, Applied Scientific Research, A 2-3, 151-181

3. Carslaw H.S., Jaeger J.C., 1959, Conduction of Heat in Solids, 2nd ed., Clarendon Press, Oxford

4. Chichinadze A.V., 1967, Calculation and Study of External Friction During Braking (in Russian), Nauka, Moscow

5. Chichinadze A.V., Braun E.D., Ginsburg A.G., Ignat'eva Z.V., 1979, Calculation, Test and Selection of Frictional Couples (in Russian), Nauka, Moscow

6. Evtushenko A., Kutsei M., 2006, Initiating of thermal cracking of materials by frictional heating, Journal of Friction and Wear, 27, 2, 9-16

7. FAzekAs G.A.G., 1953, Temperature gradients and heat stresses in brake drums, SAE Transactions, 61, 279-284

8. Jewtuszenko O., Kuciej M., Tolstoj-Sienkiewicz J., 2015, Transients and Quasi-Stationary Friction Temperature Fields (in Polish), Publ. Office of Bialystok Technical University, Bialystok

9. Kuciej M., 2012, Analytical Models of Transient Frictional Heating (in Polish), Publ. Office of Bialystok Technical University, Bialystok

10. Ling F.F., 1973, Surface Mechanics, New York, John Wiley

11. Newcomb T.P., Spurr R.T., 1967, Braking of Road Vehicles, Chapman and Hall, London

12. Noda N., Hetnarski R.B., Tanigawa Y., 2000, Thermal Stresses, Lastran Corp., Rochester, NY, USA

13. Prudnikov A.P., Brychkov Yu.A., Marichev O.I., 1986, Integrals and Series, Vol. 1. Elementary Functions, Gordon and Breach, New York

14. Prudnikov A.P., Brychkov Yu.A., Marichev O.I., 1998, Integrals and Series, Vol. 2. Special Functions, Taylor and Francis, New York

15. TopczewskA K., 2017a, Frictional heating with time dependent specific power of friction, Acta Mechanica et Automatica, 11, N 2, 111-115

16. TopczewskA K., 2017b, Thermal stresses due to frictional heating with time-dependent specific power of friction, Acta Mechanica et Automatica, 11, 4, 280-284

17. Yevtushenko A., Kuciej M., 2010, Two calculation schemes for determination of the thermal stresses due to frictional heating during braking, Journal of Theoretical and Applied Mechanics, 48, 3, 605-621

18. Yevtushenko A., Kuciej M., 2012, One-dimensional thermal problem of friction during braking: The history of development and actual state, International Journal of Heat and Mass Transfer, 55, 4118-4153

19. Yevtushenko A.A., Kuciej M., Topczewska K., 2017a, Analytical model for investigation of the effect of friction power on temperature in the disk brake, Advances in Mechanical Engineering, 9, $1-12$

20. Yevtushenko A.A., Kuciej M., Topczewska K., 2017b, Effect of the temporal profile of the friction power on thermal stresses during braking, Proceedings of the 9th International Scientific Conference "BALTTRIB 2017", Kaunas, Lithuania 123-128, https://doi.org/10.15544/balttrib.2017.23

21. Yevtushenko A.A., Kuciej M., Yevtushenko O.O., 2011, Temperature and thermal stresses in material of a pad during braking, Archive of Applied Mechanics, 81, 6, 715-726 